\title{
A remark on gradients of harmonic functions
}

Wensheng Wang

Abstract. In any $C^{1, s}$ domain, there is nonzero harmonic function $C^{1}$ continuous up to the boundary such that the function and its gradient on the boundary vanish on a set of positive measure.

\section{Introduction.}

In this note, we will extend Bourgain and Wolff's result in [2] into the general $C^{1, s}$ domain of $\mathbb{R}^{d}$.

Theorem. If $D$ is a $C^{1, s}$ domain in $\mathbb{R}^{d}$ with $s>0$ and $d \geq 3$, then there is a harmonic function $u: D \rightarrow \mathbb{R}$ which is $C^{1}$ up to the boundary and such that

$$
|\{Q \in \partial D: u(Q)=0, \nabla u(Q)=0\}|>0 .
$$

The idea for the proof of this theorem follows from the argument in [2]. We also need to use Aleksandrov-Kargaev function (see [1]) as our basic constructing factor. Since there is no reason to apply directly Alexandrov's result to the arbitrary domain, we have to work on the "almost flat" domain first and then get our final result by Kelvin transformation. 
Notation. Let $A$ and $M$ be positive large numbers. Let $\Phi_{M}$ be a collection of $C^{1, s}$ domains in $\mathbb{R}^{d}$ which are of the form

$$
\Omega=\left\{X=\left(x, x_{d}\right) \in \mathbb{R}^{d}: x_{d}>\varphi(x), x \in \mathbb{R}^{d-1}\right\}
$$

such that $\varphi$ is some $C^{1, s}$ function on $\mathbb{R}^{d-1}$ satisfying $\varphi(0)=0, \nabla \varphi(0)=$ 0 and

$$
\|\nabla \varphi\|_{\infty}+\|\nabla \varphi\|_{C^{s}} \leq e^{-M} \quad \text { and } \quad \nabla^{2} \varphi(x)=0
$$

when $|X|>1$.

When $X=\left(x, x_{d}\right) \in \partial \Omega$, we denote by $N_{X}$ the normal vector of $\partial \Omega$ at $X$, and $n_{x}$ the normal vector of $\mathbb{R}^{d-1}$ at $(x, 0)$. By the assumption of $\Omega$, we know that $\left|N_{X}-n_{x}\right| \leq e^{-M}$ for any $X=\left(x, x_{d}\right) \in \partial \Omega$. We use notation $\nabla_{T} u$ to denote the tangent gradient of $u$ on $\partial \Omega$. Finally, we usual use $B$ to denote the ball in $\mathbb{R}^{d}$ and $Q$ to denote the cube on $\mathbb{R}^{d-1}$. If $Q$ is the cube on $\mathbb{R}^{d-1}$, then $\varphi Q$ denotes its image on $\partial \Omega$ by $\varphi$. $C$ always denotes an absolute constant.

\section{Several lemmas.}

Lemma 1. Suppose $\varphi$ is a $C^{1, s}$ function on $\mathbb{R}^{d-1}$ and $\Omega=\{X=$ $\left.\left(x, x_{d}\right) \in \mathbb{R}^{d}: x_{d}>\varphi(x), x \in \mathbb{R}^{d-1}\right\}$ is a $C^{1, s}$ domain. Let $G(X, Y)$ be the Green's function of $\Omega$. Then for any $X, Y \in \partial \Omega$, we have

$$
\left|\frac{d}{d N_{X}} \frac{d}{d N_{Y}} G(X, Y)\right| \leq \frac{C}{|X-Y|^{d}}
$$

Proof. When $\Omega$ is a bounded domain, the result is known (see [4], [6]) but we do not find a good reference for the proof. When $\Omega$ is unbounded, it is not true in general. So we would like to give a proof for such special case and one will see the proof still works for bounded domains with a tiny correction.

Claim. Let $X \in \partial \Omega$ and $R>0$. If $u$ is a harmonic function in $\Omega \cap B(X, R),|u| \leq 1$ on $\Omega \cap \partial B(X, R)$ and $u=0$ on $\partial \Omega \cap B(X, R)$, then $|\nabla u(Z)| \leq C / R$ for all $Z \in \Omega \cap B(X, R / 2)$. 
Proof of Claim. We may assume that $X=0$. Let $D=\Omega \cap B(0, R)$ and $D_{-}=\Omega^{c} \cap B(0, R)$. Consider a map $\Phi: D_{-} \rightarrow D$ defined by $\Phi\left(z, z_{d}\right)=\left(z, 2 \varphi(z)-z_{d}\right)$. Then the function $u \circ \Phi$ solves

$$
\operatorname{div} A_{0} \nabla(u \circ \Phi)=0 \quad \text { in } D_{-},
$$

where

$$
A_{0}=\left(\left(\Phi^{\prime}\right)^{\perp}\right)^{-1}\left(\Phi^{\prime}\right)^{-1}=\left(\begin{array}{cc}
I & 2(\nabla \varphi)^{\perp} \\
2 \nabla \varphi & 1+4|\nabla \varphi|^{2}
\end{array}\right)
$$

is an elliptic matrix (see e.g. [3]). Let $\omega=u$ in $D, \omega=-u \circ \Phi$ in $D_{-}$. Then with $A=I$ in $D$ and $A_{0}$ in $D_{-}, \omega$ solves $\operatorname{div} A \nabla \omega=0$ in $B(0, R)$ in the weak sense, because the function $\omega$ is an odd 'reflection' of $u$. By the assumptions for the functions $u$ and $\varphi$, we know $|\omega| \leq 1$ and $\|A\|_{C^{s}} \leq C e^{-M_{1}}$. If we define functions $v(Y)=\omega(R Y)$ and $B(Y)=$ $\min \left\{R^{-s}, 1\right\} A(R Y)$ in $B(1)$, then $v$ solves $\operatorname{div} B \nabla v=0$ in $B(1)$ and $B$ has uniform $C^{s}$ bound. So by [4, Lemma 3.1], we have $|\nabla v(Y)| \leq C$. Hence, $|\nabla \omega(Z)| \leq C / R$. This proves the claim.

Now let us fix $X, Y \in \Omega$ and let $R=|X-Y|$. If we apply the claim to the function $R^{d-2} G\left(Z_{1}, Z_{2}\right)$ for $Z_{2} \in B(Y, R / 100)$ with $Z_{1} \in$ $B(X, R / 100)$ fixed, then we have $\left|\nabla_{Z_{2}} G\left(Z_{1}, Z_{2}\right)\right| \leq C / R^{d-1}$ for all $Z_{1} \in B(X, R / 100)$ and $Z_{2} \in B(Y, R / 100)$. Similarly, if we apply the claim to the function $R^{d-1} \nabla_{Z_{2}} G\left(Z_{1}, Z_{2}\right)$ for $Z_{1} \in B(X, R / 100)$ with $Z_{2} \in B(Y, R / 100)$ fixed, then we get $\left|\nabla_{Z_{1}} \nabla_{Z_{2}} G\left(Z_{1}, Z_{2}\right)\right| \leq C / R^{d}$ for all $Z_{1} \in B(X, R / 100)$ and $Z_{2} \in B(Y, R / 100)$. Finally let $Z_{1}$ go to $X$ and let $Z_{2}$ go to $Y$, to conclude.

Lemma 2. Let $\Omega$ be as in Lemma 1 with $0 \in \partial \Omega$. Suppose $u$ is a harmonic function in $\Omega$ which is $C^{1, s}$ up to the boundary. Assume the restriction function of $u$ onto $\partial \Omega$ is supported in $\partial \Omega \cap B(0,1)$. Then for all $X \in \partial \Omega$ with $|X| \leq 2$,

$$
\left|\frac{d}{d N_{X}} u(X)\right| \leq C\|u\|_{C^{1, s}(\partial \Omega)} .
$$

When $X \in \partial \Omega$ with $|X| \geq 2$, we have

$$
\left|\frac{d}{d N_{X}} u(X)\right| \leq C|X|^{-d}\|u\|_{C(\partial \Omega)} .
$$


Proof. The first result is a well known fact when $\Omega$ is either a bounded domain or the upper half space. Here we would like to give a short proof without using layer potential theory. Since $u(Y)$ has compact support on $\partial \Omega$, for $X \in \Omega$,

$$
u(X)=\int_{\partial \Omega} \frac{d}{d N_{Y}} G(X, Y) u(Y) d \sigma(Y)
$$

If $X \in \partial \Omega$ with $|X| \geq 2$, then by (1) we have

$$
\begin{aligned}
\left|\frac{d}{d N_{X}} u(X)\right| & =\left|\int_{\partial \Omega} \frac{d^{2}}{d N_{X} d Z_{Y}} G(X, Y) u(Y) d \sigma(Y)\right| \\
& \leq C\|u\|_{C(\partial \Omega)} \int_{Y \in \partial \Omega,|Y| \leq 1} \frac{1}{|X-Y|^{d}} d \sigma(Y) \\
& \leq C|X|^{-d}\|u\|_{C(\partial \Omega)} .
\end{aligned}
$$

This proves (3). Now let us fix $X \in \partial \Omega$ with $|X| \leq 2$. Choose a function $\phi \in C_{0}^{\infty}\left(\mathbb{R}^{d}\right)$ with $\phi(Z)=1$ when $|Z| \leq 10$ and $\phi(Z)=0$ when $|Z| \geq 20$. Let us write

$$
u_{\phi}(Y)=u(Y)-u(X) \phi(Y-X)-\left\langle\nabla_{T} u(X), Y-X\right\rangle \phi(Y-X),
$$

which is supported in $\partial \Omega \cap\{|Y| \leq 15\}$ and bounded by

$$
\left\|\nabla_{T} u\right\|_{C^{s}(\partial \Omega)}|Y-X|^{1+s}, \quad \text { when } \quad|Y-X| \leq 8
$$

So we have

$$
\begin{aligned}
u(Z)= & \int_{\partial \Omega} \frac{d}{d N_{Y}} G(Z, Y) u_{\phi}(Y) d \sigma(Y) \\
& +u(X) \int_{\partial \Omega} \frac{d}{d N_{Y}} G(Z, Y) \phi(Y-X) d \sigma(Y) \\
& +\int_{\partial \Omega} \frac{d}{d N_{Y}} G(Z, Y)\left\langle\nabla_{T} u(X), Y-X\right\rangle \phi(Y-X) d \sigma(Y) \\
= & u_{1}(Z)+u_{2}(Z)+u_{3}(Z)
\end{aligned}
$$


For $u_{1}$,

$$
\begin{aligned}
\left|\frac{d}{d N_{X}} u_{1}(X)\right|= & \left|\int_{\partial \Omega} \frac{d^{2} G(X, Y)}{d N_{X} d N_{Y}} u_{\phi}(Y) d \sigma(Y)\right| \\
\leq & C \int_{X \in \partial \Omega,|X-Y|<8} \frac{|X-Y|^{1+s}}{|X-Y|^{d}}\left\|\nabla_{T} u\right\|_{C^{s}(\partial \Omega)} d \sigma(Y) \\
& +C \int_{X \in \partial \Omega,|X-Y| \geq 8} \frac{1}{|X-Y|^{d}}\|u\|_{C^{1}(\partial \Omega)} \\
\leq & C\|u\|_{C^{1, s}(\partial \Omega)} .
\end{aligned}
$$

Notice that $u_{2}$ is a bounded harmonic function whose boundary value is 1 on $\partial \Omega \cap B(0,4)$. So apply the claim in Lemma 1 to the function $u(X)-u_{2}(\cdot)$, we have $\left|\nabla u_{2}(Z)\right| \leq C\|u\|_{\infty}$ for $Z \in \Omega \cap B(0,2)$ so that

$$
\left|\frac{d}{d N_{X}} u_{2}(X)\right| \leq C\|u\|_{C^{1, s}(\partial \Omega)} .
$$

Finally consider the harmonic function $u_{3}(Z)-\left\langle\nabla_{T} u(X), Z-X\right\rangle$ which is bounded in $\Omega \cap B(0,4)$ and whose boundary value is zero on $\partial \Omega \cap$ $B(0,4)$. So again by using the claim in Lemma 1 , we have

$$
\left|\frac{d}{d N_{X}} u_{3}(X)\right| \leq C\|u\|_{C^{1}(\partial \Omega)} \leq C\|u\|_{C^{1, s}(\partial \Omega)},
$$

since $\left\langle\nabla_{T} u(X), Z-X\right\rangle$ is linear. This proves Lemma 2.

Now let $a$ and $\varepsilon$ be two positive numbers. Let

$$
E_{\varepsilon}^{a}(X)=-a \frac{\varepsilon+x_{d} / a}{\left|X / a+\varepsilon e_{d}\right|^{d}}
$$

We denote by $n_{x}$ the normal vector of $\mathbb{R}^{d-1}$ in $\mathbb{R}^{d}$ at $x \in \mathbb{R}^{d-1}$.

Lemma 3. We have the following properties for $E_{\varepsilon}^{a}(X)$ :

$$
\left|\nabla^{i} E_{\varepsilon}^{a}(X)\right| \leq C a^{-i+1} \min \left\{\varepsilon^{-d-i+1},\left|\frac{X}{a}\right|^{-d-i+1}\right\}
$$


for all $X \in \Omega$ and $i=0,1,2$.

$$
\begin{aligned}
& \mid \int_{\varphi Q(0, b)}\left(\left|1+\frac{d}{d N} E_{\varepsilon}^{a}(X)\right|^{p}-1\right) d \sigma(X) \\
& -\int_{Q(0, b)}\left(\left|1+\frac{d}{d n} E_{\varepsilon}^{a}(x)\right|^{p}-1\right) d x \mid \\
& \leq C e^{-p M} M^{d-1} a^{d-1}
\end{aligned}
$$

if $\varepsilon \leq e^{-M}$.

(6) $\quad \int_{\varphi Q(0, b)}\left(\left|1+\frac{d}{d N} E_{\varepsilon}^{a}(X)\right|^{p}-1\right) d \sigma(X) \leq-\left(\eta-\frac{C}{M}\right) a^{d-1}$

if $p>0$ is small but independent of $\varepsilon, M, a$. Here $b=a M$ in (5) and (6), and $\eta$ is an absolute small positive number.

Proof. (4) follows directly from the calculation. After a change of variable, the left hand side of (5) is the integral over a subset $\{|x|<b\}$ of $\mathbb{R}^{d-1}$ of the following integrand

$$
\left(\left|1+\left\langle\nabla E_{\varepsilon}^{a}(X), N_{X}\right\rangle\right|^{p}-1\right)\left(1+|\nabla \varphi(x)|^{2}\right)^{1 / 2}-\left(\left|1+\left\langle\nabla E_{\varepsilon}^{a}(x), n_{x}\right\rangle\right|^{p}-1\right) .
$$

If we introduce a term $-\left|1+\left\langle\nabla E_{\varepsilon}^{a}(x), n_{x}\right\rangle\right|^{p}\left(1+|\nabla \varphi(x)|^{2}\right)^{1 / 2}$ and subtract it, then the integrand becomes

$$
\begin{aligned}
& \left(\left|1+\left\langle\nabla E_{\varepsilon}^{a}(X), N_{X}\right\rangle\right|^{p}-\left|1+\left\langle\nabla E_{\varepsilon}^{a}(x), n_{x}\right\rangle\right|^{p}\right)\left(1+|\nabla \varphi(x)|^{2}\right)^{1 / 2} \\
& \left.+\left|1+\left\langle\nabla E_{\varepsilon}^{a}(x), n_{x}\right\rangle\right|^{p}\left(1+|\nabla \varphi(x)|^{2}\right)^{1 / 2}-1\right) \\
& \left.-\left(1+|\nabla \varphi(x)|^{2}\right)^{1 / 2}-1\right) \\
= & \mathrm{I}+\mathrm{II}+\mathrm{III} .
\end{aligned}
$$

Up to multiplying a constant, I is bounded by

$$
\begin{aligned}
\mid\left\langle\nabla E_{\varepsilon}^{a}(X),\right. & \left.N_{X}\right\rangle-\left.\left\langle\nabla E_{\varepsilon}^{a}(x), n_{x}\right\rangle\right|^{p} \\
& \leq\left|\left\langle\nabla E_{\varepsilon}^{a}(X)-\nabla E_{\varepsilon}^{a}(x), n_{x}\right\rangle\right|^{p}+\left|\left\langle\nabla E_{\varepsilon}^{a}(X), N_{X}-n_{x}\right\rangle\right|^{p} \\
& =\mathrm{I}_{1}+\mathrm{I}_{2} .
\end{aligned}
$$

For $\mathrm{I}_{1}$, since $X=(x, \varphi(x))$, by $(4)$,

$$
\mathrm{I}_{1}=\left|\left\langle\nabla E_{\varepsilon}^{a}(X)-\nabla E_{\varepsilon}^{a}(x), n_{x}\right\rangle\right|
$$




$$
\begin{aligned}
& \leq a^{-2+1}\left|\frac{x}{a}\right|^{-d-2+1}|X-x| \\
& \leq a^{-1}\left|\frac{x}{a}\right|^{-d-1}|\varphi(x)| \\
& \leq e^{-M}\left|\frac{x}{a}\right|^{-d} .
\end{aligned}
$$

So

$$
\int_{|x| \leq b}\left|\mathrm{I}_{1}\right| d x \leq M^{d(1-p)-1} e^{-p M} a^{d-1} .
$$

Since $\left|N_{X}-n_{x}\right| \leq C\|\nabla \varphi\|_{\infty} \leq C e^{-M}$, again by (4),

$$
\int_{|x| \leq b}\left|\mathrm{I}_{2}\right| d x \leq C e^{-p M} \int_{|x| \leq b}\left|\frac{x}{a}\right|^{-d p} d x=C M^{d(1-p)-1} e^{-p M} a^{d-1} .
$$

By a similar method, using (4) and the assumption of $\varphi$,

$$
\begin{aligned}
\int_{|x| \leq b}|\mathrm{II}| d x & \leq C \int_{|x| \leq b}\left(1+\left|\frac{x}{a}\right|^{-d}\right)^{p} e^{-M} d x \\
& \leq C a^{d-1} e^{-M} M^{d(1-p)-1} .
\end{aligned}
$$

And it is trivial to get

$$
\int_{|x| \leq b}|\mathrm{III}| d x \leq C e^{-M} \int_{|x| \leq b} d x \leq C e^{-M} a^{d-1} M^{d-1} .
$$

So combining those estimates of the integrations for $\mathrm{I}=\mathrm{I}_{1}+\mathrm{I}_{2}$, II and III, we have that the left hand side of (4) is bounded by

$$
\begin{aligned}
C a^{d-1}\left(M^{d(1-p)-1} e^{-p M}+M^{d(1-p)-1} e^{-p M}\right. & \left.+M^{d-1} e^{-M}\right) \\
& \leq C a^{d-1} M^{d-1} e^{-p M} .
\end{aligned}
$$

Let us now prove (6). From [1] or [2], we know that if $p>0$ is small enough then for all small $\varepsilon$,

$$
\int_{\mathbb{R}^{d-1}}\left(\left|1+\frac{d}{d n} E_{\varepsilon}^{a}(x)\right|^{p}-1\right) d x \leq-\eta a^{d-1},
$$

with a small absolute positive number $\eta$. From this integral, we easily get

$$
\int_{|x|<b}\left(\left|1+\frac{d}{d n} E_{\varepsilon}^{a}(x)\right|^{p}-1\right) d x
$$




$$
\begin{aligned}
& \leq-\eta a^{d-1}+\int_{|x|>b}|| 1+\left.\frac{d}{d n} E_{\varepsilon}^{a}(x)\right|^{p}-1 \mid d x \\
& \leq-\eta a^{d-1}+C \int_{|x|>b}\left|\frac{x}{a}\right|^{-d-1} d x \\
& \leq-\left(\eta-C M^{-2}\right) a^{d-1}
\end{aligned}
$$

by (4). So combine (7) and (5). We have that the left hand side of (6) is less than or equal to

$$
-\left(\eta-C M^{-2}\right) a^{d-1}+C e^{-p M} M^{d-1} a^{d-1}-\left(\eta-\frac{C}{M}\right) a^{d-1}
$$

when $M$ is large enough independently of $a$ and $\varepsilon$.

We state our main lemma.

Lemma 4. If $p>0$ and $1 / M>0$ are small enough, then for any $\Omega \in \Phi_{M}$ and $\varepsilon>0$ and any cube $Q=Q(0, l)$ on $\mathbb{R}^{d-1}$ with $l<1$ there exists a harmonic function $F_{\varepsilon}^{Q}$ which is $C^{1}$ up to $\bar{\Omega}$ with $\left.\operatorname{supp} F_{\varepsilon}^{Q}\right|_{\partial \Omega} \subset$ $\varphi B(0$, cl $)$ such that

$$
\int_{\varphi Q}\left|1+\frac{d}{d N} F_{\varepsilon}^{Q}(X)\right|^{p} d \sigma(X) \leq e^{-2 \beta p}|\varphi Q|,
$$

(9) $\left|\nabla F_{\varepsilon}^{Q}(X)\right| \leq C \min \left\{\varepsilon^{-d}, e^{-(d-1 / 2) M}\left(\left|\frac{X}{l}\right|^{-d}+\left|\frac{X}{l}\right|^{-d+1 / 2}\right)\right\}$,

if $X \in \partial \Omega$, where $\beta>0$ is such that

$$
e^{-2 \beta p}=1-\frac{\eta}{2} e^{-(d-1) M} .
$$

Proof. Let $a=e^{-M} l$ and $E_{\varepsilon}^{a}(X)$ be as above. Define

$$
I_{j}=\left\{x \in \mathbb{R}^{d-1}: 2^{j-1} l \leq|x|<2^{j+1} l\right\}
$$

and $\rho_{j}$ a cut-off function in $\mathbb{R}^{d}$ with supp $\rho_{j} \subset Q\left(0,2^{j+1} l\right) \backslash Q\left(0,2^{j-1} l\right)$ and $\left|\nabla^{i} \rho_{j}\right| \leq 1 /\left(2^{j} l\right)^{i}$, for $i=0,1,2$ and for $j=0,1, \ldots$ such that $\sum_{j} \rho_{j}(X)=1$ for $|X|>1$. Let $\rho=1-\sum \rho_{j}(X)$. Denote by $F_{\varepsilon}^{Q}$ the harmonic extension into $\Omega$ of $\left.\rho E_{\varepsilon}^{a}(X)\right|_{\partial \Omega}$ and $Q_{j, \varepsilon}^{a}$ the harmonic 
extension into $\Omega$ of $\left.\rho_{j} E_{\varepsilon}^{a}(X)\right|_{\partial \Omega}$. Let $Q_{\varepsilon}^{a}=\sum_{j} Q_{j, \varepsilon}^{a}$. Then $E_{\varepsilon}^{a}=$ $F_{\varepsilon}^{a}+Q_{\varepsilon}^{a}$.

An easy computation and (4) of Lemma 3 imply that

$$
\begin{aligned}
\left\|Q_{j, \varepsilon}^{a}\right\|_{\infty} & \leq C a^{d}\left(2^{j} l\right)^{-d+1}, \\
\left\|\nabla_{T} Q_{j, \varepsilon}^{a}\right\|_{\infty} & \leq C a^{d}\left(2^{j} l\right)^{-d}, \\
\left\|\nabla_{T} Q_{j, \varepsilon}^{a}\right\|_{C^{s}} & \leq C a^{d}\left(2^{j} l\right)^{-d-s} .
\end{aligned}
$$

If we let $u(Y)=Q_{j, \varepsilon}^{a}\left(2^{j} l Y\right)$, then the restriction function of $u$ onto $\partial \Omega$ is supported in $\partial \Omega \cap B(0,1)$ and $\|u\|_{C^{1, s}} \leq C a^{d}\left(2^{j} l\right)^{-d+1}$ by the above estimates. So apply Lemma 2 to $u$. When $X \in \partial \Omega$ with $|X| \leq 2^{j+2} l$, we get

$$
\left|\frac{d}{d N_{X}} Q_{j, \varepsilon}^{a}(X)\right| \leq C a^{d}\left(2^{j} l\right)^{-d}
$$

When $X \in \partial \Omega$ with $|X|>2^{j+2} l$,

$$
\left|\frac{d}{d N_{X}} Q_{j, \varepsilon}^{a}(X)\right| \leq C a^{d}|X|^{-d} \leq C a^{d}|X|^{-d+1 / 2}\left(2^{j} l\right)^{-1 / 2} .
$$

Hence

$$
\begin{aligned}
\left|\frac{d}{d N} Q_{\varepsilon}^{a}(X)\right| & \leq\left(\sum_{|X|<2^{j+2} l}+\sum_{|X|>2^{j+2} l}\right)\left|\frac{d}{d N} Q_{j, \varepsilon}^{a}(X)\right| \\
& \leq C \sum_{|X|<2^{j+2} l} a^{d}\left(2^{j} l\right)^{-d}+C \sum_{|X|>2^{j+2} l} a^{d}\left(2^{j} l\right)^{-1 / 2}|X|^{-d+1 / 2} \\
& \leq C a^{d}|X|^{-d}+C a^{d}|X|^{-d+1 / 2} l^{-1 / 2} \\
& \leq C\left(\left|\frac{X}{a}\right|^{-d}+\left|\frac{X}{a}\right|^{-d+1 / 2}\right),
\end{aligned}
$$

since $a=e^{-M} l$. We notice that if $|X| \leq 4 l$, the process of estimates above also give

$$
\left|\frac{d}{d N} Q_{\varepsilon}^{a}(X)\right| \leq C e^{-M}
$$


Now we estimate (7). Let $b=M a$.

$$
\begin{aligned}
\int_{\varphi Q}(\mid 1+ & \left.\left.\frac{d}{d N} F_{\varepsilon}^{Q}(X)\right|^{p}-1\right) d \sigma(X) \\
= & \int_{\varphi Q(0, b)}\left(\left|1+\frac{d}{d N}\left(E_{\varepsilon}^{a}(X)-Q_{\varepsilon}^{a}(X)\right)\right|^{p}-1\right) d \sigma(X) \\
& +\int_{\varphi Q \backslash \varphi Q(0, b)}\left(\left|1+\frac{d}{d N} F_{\varepsilon}^{Q}(X)\right|^{p}-1\right) d \sigma(X) \\
\leq & \int_{\varphi Q(0, b)}\left(\left|1+\frac{d}{d N} E_{\varepsilon}^{a}(X)\right|^{p}-1\right) d \sigma(X) \\
& +\int_{\varphi Q(0, b)}\left(\left|1+\frac{d}{d N} Q_{\varepsilon}^{a}(X)\right|^{p}-1\right) d \sigma(X) \\
& +\int_{\varphi Q \backslash \varphi Q(0, b)}\left(\left|1+\frac{d}{d N} F_{\varepsilon}^{Q}(X)\right|^{p}-1\right) d \sigma(X)
\end{aligned}
$$

by triangle inequality. When $|x|>b$, the integrand in the last integral is bounded by $C|x / a|^{-d / 2}$, by (4) and (11). So

$$
\int_{\varphi Q \backslash \varphi Q(0, b)}\left(\left|1+\frac{d}{d N} F_{\varepsilon}^{Q}(X)\right|^{p}-1\right) d \sigma(X) \leq C a^{d-1} M^{-1 / 2}
$$

Since $p<1$, by Hölder inequality,

$$
\begin{aligned}
\int_{\varphi Q(0, b)} & \left|\frac{d}{d N} Q_{\varepsilon}^{a}(X)\right|^{p} d \sigma \\
& \leq \sum_{j} \int_{\varphi Q(0, b)}\left|\frac{d}{d N} Q_{j, \varepsilon}^{a}(X)\right|^{p} d \sigma \\
& \leq C b^{(1-p / 2)(d-1)} \sum_{j}\left(\int_{\varphi Q(0, b)}\left|\frac{d}{d N} Q_{j, \varepsilon}^{a}(X)\right|^{2} d \sigma\right)^{p / 2} .
\end{aligned}
$$

Notice that $\varphi \Omega$ is a Lipschitz graph with uniform bound. Theorem 2.2.6 of [5] shows that

$$
\int_{\varphi \Omega}\left|\frac{d}{d N} Q_{j, \varepsilon}^{a}\right|^{2} d \sigma \leq C \int_{\varphi \Omega}\left|\nabla_{T} Q_{j, \varepsilon}^{a}\right|^{2} d \sigma .
$$


So by (10),

$$
\begin{aligned}
\int_{\varphi Q(0, b)} \mid \frac{d}{d N} & \left.Q_{\varepsilon}^{a}(X)\right|^{p} d \sigma \\
& \leq C b^{(1-p / 2)(d-1)} \sum_{j}\left(\int_{\varphi \Omega}\left|\nabla_{T} Q_{j, \varepsilon}^{a}\right|^{2} d \sigma\right)^{p / 2} \\
& \leq C b^{(1-p / 2)(d-1)} \sum_{j}\left(\int_{\varphi I_{j}}\left|a^{d}\left(2^{j} l\right)^{-d}\right|^{2} d \sigma\right)^{p / 2} \\
& \leq C a^{d p} b^{(1-p / 2)(d-1)} \sum_{j}\left(2^{j} l\right)^{-d p+p(d-1) / 2} \\
& \leq C a^{d-1}(a l)^{p(d+1) / 2} M^{(1-p / 2)(d-1)} \\
& =C a^{d-1} e^{-p M(d+1) / 2} M^{(1-p / 2)(d-1)} .
\end{aligned}
$$

Now apply (6) to the first term in the left hand side of (13) and combine (13), (14), and (15). The left hand side of (13) is less than or equal to

$$
\begin{aligned}
-\left(\eta-\frac{C}{M}\right) a^{d-1}+C a^{d-1} M^{-1 / 2} & +C a^{d-1} e^{-p M(d+1) / 2} M^{(1-p / 2)(d-1)} \\
& \leq-\frac{\eta}{2} a^{d-1}=-\frac{\eta}{2} e^{-M(d-1)}|\varphi Q|
\end{aligned}
$$

Hence

$$
\int_{\varphi Q}\left|1+\frac{d}{d N} F_{\varepsilon}^{Q}(X)\right|^{p} d \sigma \leq\left(1-\frac{\eta}{2} e^{-M(d-1)}\right)|\varphi Q|=e^{-2 \beta p}|\varphi Q|,
$$

with some $\beta>0$.

Now we turn to prove (8). First we have

$$
\begin{aligned}
\left|\nabla F_{\varepsilon}^{a}(X)\right| & \leq\left|\nabla Q_{\varepsilon}^{a}(X)\right|+\left|\nabla E_{\varepsilon}^{a}(X)\right| \\
& \leq C\left(\left|\frac{X}{a}\right|^{-d}+\left|\frac{X}{a}\right|^{-d+1 / 2}\right) \\
& \leq C e^{-M(d-1 / 2)}\left(\left|\frac{X}{l}\right|^{-d}+\left|\frac{X}{l}\right|^{-d+1 / 2}\right)
\end{aligned}
$$

by (4) and (11). In order to bound $\left|\nabla F_{\varepsilon}^{a}(X)\right|$ by $C \varepsilon^{-d}$, we notice that when $|X|<l \varepsilon<l$,

$$
\left|\frac{d}{d N} Q_{\varepsilon}^{a}(X)\right| \leq C
$$


is bounded by (12) and of course is less than $C \varepsilon^{-d}$. The remaining part follows directly from calculation and (4).

Corollary 5. Let $p, M$ and $\varepsilon$ be as in Lemma 4 . Let $\Omega \in \Phi_{2 M}$. For any $X_{Q}=\left(c_{Q}, \varphi\left(c_{Q}\right)\right) \in \partial \Omega$ and $s_{0}>0, Q \subset B\left(c_{Q}, s_{0}\right)$ is a cube in $\mathbb{R}^{d-1}$. For any function $I: \partial \Omega \rightarrow \mathbb{R}$ with $\operatorname{supp} I \subset \varphi \Omega$ such that

$$
\left|I\left(X_{Q}\right)^{-1}\right|\left\|I(\cdot)-I\left(X_{Q}\right)\right\|_{\infty} \leq \frac{1}{8} \eta e^{-M(d-1)},
$$

there exists a harmonic function $F_{\varepsilon}^{Q}$ in $\Omega$ which is $C^{1}$ up to $\bar{\Omega}$ such that $\operatorname{supp} F_{\varepsilon}^{Q} \subset \varphi B\left(c_{Q}, \varepsilon l(Q)\right)$ and

$$
\begin{aligned}
& \int_{\varphi Q}\left|I(X)+I\left(X_{Q}\right) \frac{d F_{\varepsilon}^{Q}}{d N}(X)\right|^{p} d \sigma(X) \leq e^{-2 \beta p}\left|I\left(X_{Q}\right)\right|^{p}|\varphi Q| \\
& \left|\nabla F_{\varepsilon}^{Q}(X)\right| \\
& \leq C \min \left\{\varepsilon^{-d}, e^{-M(d-1 / 2)}\left(\left|\frac{X-X_{Q}}{l(Q)}\right|^{-d}+\left|\frac{X-X_{Q}}{l(Q)}\right|^{-d+1 / 2}\right)\right\}
\end{aligned}
$$

Proof. Under a new coordinate system such that $X_{Q}$ is the new origin and the tangent space of $\partial \Omega$ at $X_{Q}$ is the new $\mathbb{R}^{d-1}, \Omega \in \Phi_{M}$. Then this corollary follows directly from Lemma 4 .

Let us first prove a weaker version of our theorem.

Theorem 1. Let $\Omega$ be as in the Corollary 5. Then for any small number $s_{0}>0$, there is a harmonic function $u: \Omega \rightarrow \mathbb{R}$ which is $C^{1}$ up to $\bar{\Omega}$ and such that

$$
\left|\left\{X \in \partial \Omega:|X| \leq s_{0}, u(X)=0, \nabla u(X)=0\right\}\right|>0 .
$$

We will give the recursive construction. Let $Q_{0}=Q_{0}\left(0, s_{0}\right) \subset$ $\mathbb{R}^{d-1}$. Let $\left\{\delta_{n}\right\}_{1}^{\infty}$ be a sequence of small numbers such that $\delta_{n}^{-1} \in$ $\mathbb{Z}$ which are chosen by induction later. Let $\left\{K_{n}\right\}$ and $\left\{\varepsilon_{n}\right\}$ be two sequences of numbers which are decided later with $K_{n} \nearrow+\infty$ and $\varepsilon \searrow 0 . a$ will be a large universal number also decided later. 
Let $\Re_{n}$ be the collection of $\delta_{n}^{-(d-1)}$ cubes of side $\delta_{n}$ in $\mathbb{R}^{d-1}$ whose union is $Q_{0}$. Let $\Im_{n}$ be a subset of $\Re_{n}$ such that

$$
\left(\int_{\varphi V_{n}}\left|\frac{d u_{n}}{d N}\right|^{p} d \sigma(X)\right)^{1 / p} \leq A e^{-\beta n},
$$

where $V_{n}=\bigcup\left\{Q: Q \in \Im_{n}\right\}$ and $u_{n}$ is the $C^{1, s}$ function in $\Omega$ defined by induction later. When $n=1$, let $\delta_{0}=s_{0}$ and $\Im_{0}=\left\{Q_{0}\right\}$ and $u_{0}$ a harmonic function in $\Omega$ such that $\left.u_{0}\right|_{\partial \Omega} \in C_{0}^{1, s}\left(\varphi Q_{0} / 1000\right)$. Suppose we have $\Im_{n}$ and $u_{n}$. Let $\delta_{n+1}$ with $\delta_{n+1} / \delta_{n} \in \mathbb{Z}$ be such that $\delta_{n+1}$ small enough and decided in the following lemmas. Let $\Im_{n+1} \subset \Re_{n+1}$ be such that $Q \in \Im_{n+1}$ satisfies

$$
\begin{gathered}
Q^{\prime} \in \Im_{n} \text { for all } Q^{\prime} \in \Re_{n} \text { with } Q \subset Q^{\prime}, \\
\left(\frac{1}{|\varphi Q|} \int_{\varphi Q}\left|\frac{d u_{n}}{d N}\right|^{p} d \sigma(X)\right)^{1 / p} \leq K_{n+1} e^{-\beta n} .
\end{gathered}
$$

Now let us define

$$
u_{n+1}(X)=u_{n}(X)+\sum_{Q \in \Im_{n+1}} \frac{d u_{n}}{d N}\left(X_{Q}\right) F_{\varepsilon_{n+1}}^{Q}(X),
$$

where $F_{\varepsilon_{n+1}}^{Q}$ is as in Corollary 5 .

Lemma 6. For $X \in \partial \Omega$,

$$
\sum_{\substack{Q \in \Im_{n+1} \\\left|x-c_{Q}\right|>\rho}}\left|\nabla F_{\varepsilon_{n+1}}^{Q}(X)\right| \leq C e^{-M(d-1 / 2)}\left(\frac{\delta_{n+1}}{\rho}+\left(\frac{\delta_{n+1}}{\rho}\right)^{1 / 2}\right),
$$

if $\rho>C \delta_{n+1}$.

$$
\left|\nabla u_{n+1}(X)-\nabla u_{n}(X)\right| \leq C K_{n+1} e^{-\beta n} \varepsilon_{n+1}^{-d},
$$

if $\delta_{n+1}$ small enough.

Proof. By (17) of Corollary 5, the left hand side of (21) is bounded by

$$
\begin{array}{r}
\sum_{|x-c|_{Q}>\rho} e^{-M(d-1 / 2)}\left(\left|\frac{X-c_{Q}}{\delta_{n+1}}\right|^{-d}+\left|\frac{X-c_{Q}}{\delta_{n+1}}\right|^{-d+1 / 2}\right) \\
\leq C e^{-M(d-1 / 2)}\left(\frac{\delta_{n+1}}{\rho}+\left(\frac{\delta_{n+1}}{\rho}\right)^{1 / 2}\right),
\end{array}
$$


if $\rho>C \delta_{n+1}$. Let us prove (22) now. Since $d u_{n} / d N$ is continuous, after making $\delta_{n+1}$ small (20) will imply that

$$
\left|\frac{d u_{n}}{d N}\left(X_{Q^{\prime}}\right)\right| \leq 2 K_{n+1} e^{-\beta n}
$$

for all $Q^{\prime} \in \Im_{n+1}$. For $X \in \partial \Omega$, there is at most one $Q \in \Im_{n+1}$ such that for any other $Q^{\prime} \neq Q, Q^{\prime} \in \Im_{n+1},\left|x-c_{Q^{\prime}}\right|>\rho$. Then the left hand side of (15) is bounded by

$$
\begin{aligned}
\sum_{\substack{Q^{\prime} \in \Im_{n+1} \\
Q^{\prime} \neq Q}}\left|\frac{d u_{n}}{d N}\left(X_{Q^{\prime}}\right)\right|\left|\nabla F_{\varepsilon_{n+1}}^{Q^{\prime}}(X)\right|+\mid & \frac{d u_{n}}{d N}\left(X_{Q}\right)|| \nabla F_{\varepsilon_{n+1}}^{Q}(X) \mid \\
& \leq 2 K_{n+1} e^{-\beta n}\left(C+C \varepsilon_{n+1}^{-d}\right) \\
& \leq C K_{n+1} e^{-\beta n} \varepsilon_{n+1}^{-d}
\end{aligned}
$$

where we used (21) and (17) in the first inequality.

The following lemma says our process in construction of $u_{n}$ is possible, i.e. (18) is true.

Lemma 7. There exists a large universal constant $A$ such that

$$
\left(\int_{\varphi V_{n+1}}\left|\frac{d u_{n+1}}{d N}\right|^{p} d \sigma\right)^{1 / p} \leq A e^{-\beta(n+1)} .
$$

Proof. As in [2], we first state a claim as follows. One may find the proof in [2].

Claim. If $\delta_{n+1}$ is small enough, then for all $X \in \varphi Q, Q \in \Im_{n+1}$,

$$
\begin{aligned}
& \sum_{\substack{Q^{\prime} \in \Im_{n+1} \\
Q^{\prime} \neq Q}}\left|\frac{d u_{n}}{d N}\left(X_{Q^{\prime}}\right)\right|\left|\nabla F_{\varepsilon_{n+1}}^{Q^{\prime}}(X)\right| \\
& \quad \leq C e^{-M(d-1 / 2)}\left|\frac{d u_{n}}{d N}(X)\right|+e^{-M(d-1 / 2)} e^{-4 \beta(n+1)} .
\end{aligned}
$$

We would like to point out the idea. Divide the sum into two parts: $\sum_{\delta_{n+1}<\left|X_{Q}-X\right|<L \delta_{n+1}}$ and $\sum_{\left|X_{Q}-X\right| \geq L \delta_{n+1}}$. For the first term, use (21) 
and notice that if $\delta_{n+1}$ is small then the numbers $d u_{n}\left(X_{Q^{\prime}}\right) / d N$ are close to $d u_{n}(X) / d N$ up to an error term. For the second term, when $n$ fixed, $d u_{n}\left(X_{Q^{\prime}}\right) / d N$ are bounded uniformly in $Q^{\prime} \neq Q$. For the remaining part, apply (21) again and let $L$ be big.

Now let us take

$$
I=\left\{Q \in \Im_{n+1}:\left|\frac{d u_{n}}{d N}\left(X_{Q}\right)\right|>e^{-4 \beta(n+1)}\right\}
$$

and

$$
\Pi=\Im_{n+1} \backslash I
$$

Let $X \in \varphi Q$ for some $Q \in \Im_{n+1}$. Denote

$$
J(X)=\frac{d u_{n}}{d N}(X)+\sum_{\substack{Q^{\prime} \in \Im_{n+1} \\ Q^{\prime} \neq Q}} \frac{d u_{n}}{d N}\left(X_{Q^{\prime}}\right) \frac{d}{d N} F_{\varepsilon_{n+1}}^{Q^{\prime}}(X)
$$

Then

$$
\frac{d u_{n+1}}{d N}(X)=J(X)+\frac{d u_{n}}{d N}\left(X_{Q}\right) \frac{d}{d N} F_{\varepsilon_{n+1}}^{Q}(X)
$$

by the definition of $u_{n+1}$. If $Q \in I$, then after making $\delta_{n+1}$ small,

$$
\begin{aligned}
\left|J(X)-\frac{d u_{n}}{d N}\left(X_{Q}\right)\right| & \leq C e^{-M(d-1 / 2)}\left|\frac{d u_{n}}{d N}\left(X_{Q}\right)\right| \\
& \leq \frac{1}{8} \eta e^{-M(d-1)}\left|\frac{d u_{n}}{d N}\left(X_{Q}\right)\right|
\end{aligned}
$$

by claim and by letting $M$ large. So apply Corollary 5 ,

$$
\begin{aligned}
\int_{\varphi Q}\left|\frac{d u_{n+1}}{d N}(X)\right|^{p} d \sigma(X) & \leq e^{-2 \beta p}|\varphi Q|\left|\frac{d u_{n}}{d N}\left(X_{Q}\right)\right|^{p} \\
& \leq e^{-3 \beta p / 2} \int_{\varphi Q}\left|\frac{d u_{n}}{d N}\right|^{p} d \sigma(X)
\end{aligned}
$$

if $\delta_{n+1}$ small again. When $Q \in \Pi$, write

$$
J=\frac{d u_{n}}{d N}\left(X_{Q}\right)+J_{1}, \quad \text { where }\left|J_{1}\right| \leq C e^{-M(d-1 / 2)} e^{-4 \beta(n+1)}
$$


by claim. So apply Corollary 5 again,

$$
\begin{aligned}
\int_{\varphi Q} \mid \frac{d u_{n+1}}{d N} & \left.(X)\right|^{p} d \sigma(X) \\
& \leq\left(e^{-2 p \beta} e^{-4 \beta(n+1) p}+C e^{-4 \beta(n+1) p}\right)|\varphi Q| \\
& \leq C e^{-4 \beta(n+1) p}|\varphi Q|
\end{aligned}
$$

Combine (23) and (24). By induction, we have

$$
\begin{aligned}
\int_{\varphi V_{n+1}}\left|\frac{d u_{n+1}}{d N}\right|^{p} & \leq e^{-3 p \beta / 2} \int_{\substack{\varphi Q \\
Q \in V_{n+1} \cap I}}\left|\frac{d u_{n}}{d N}\right|^{p}+C e^{-4 \beta(n+1) p} \sum_{Q \in V_{n+1} \cap \Pi}|\varphi Q| \\
& \leq A^{p}\left(e^{-p \beta / 2}+C A^{-p} e^{-3 p(n+1) \beta}\right) e^{-p \beta(n+1)} \\
& \leq A^{p} e^{p \beta(n+1)},
\end{aligned}
$$

if $A$ is large and independent of $n$.

Proof of Theorem 1. Choose $K_{n}$ and $\varepsilon_{n}$ such that

$$
\sum \varepsilon_{n+1}^{-d} K_{n+1} e^{-\beta n} \leq C_{0}<+\infty
$$

and

$$
\sum K_{n+1}^{-p}+\varepsilon_{n+1}^{d-1} \leq \frac{s_{0}^{d-1}}{1000 C} .
$$

Then the (21) of Lemma 6 implies that

$$
\sum\left|\nabla u_{n+1}-\nabla u_{n}\right| \leq C \sum K_{n+1} \varepsilon_{n+1}^{-d} e^{-\beta n} \leq C C_{0}<+\infty
$$

i.e. $u=\sum u_{n}$ is $C^{1}$ up to $\Omega$. On the other hand, by the definition of $u$ and $u_{n+1}$,

$$
\begin{aligned}
\left|\left\{X \in \varphi Q_{0}: u(X) \neq 0\right\}\right| & \leq \sum_{n}\left|\left\{X \in \varphi Q_{0}: u_{n+1}(X) \neq u_{n}(X)\right\}\right| \\
& \leq \sum_{n} \sum_{Q \in \Im_{n+1}}\left|\varphi\left(B\left(c_{Q}\right), \varepsilon_{n+1} l(Q)\right)\right| \\
& \leq \sum_{n} \delta_{n+1}^{-(d-1)}\left(\delta_{n+1} \varepsilon_{n+1}\right)^{d-1} \\
& =C \sum_{n+1} \varepsilon_{n-1}^{d-1} \\
& \leq \frac{s_{0}^{d-1}}{1000}
\end{aligned}
$$


In order to estimate the gradient term, we notice that if a point $X \in$ $\varphi\left(Q_{0}\right)$ is in some $\varphi Q_{n}$ 's with infinite many $n$, then we know by (20) and the continuity of $d u / d N$ that $d u(X) / d N=0$. So again by (20) and (18),

$$
\begin{aligned}
\left|\left\{X \in \varphi Q_{0}: \frac{d u}{d N}(X) \neq 0\right\}\right| & \leq \sum_{n}\left|\varphi V_{n} \backslash \varphi V_{n+1}\right| \\
& =\sum_{n} \sum_{Q \in \varphi V_{n+1} \backslash \varphi V_{n}}|\varphi Q| \\
& \leq \sum_{n} K_{n+1}^{-p} e^{-\beta n p} \sum_{Q \in \varphi V_{n+1} \backslash \varphi V_{n}} \int_{\varphi Q}\left|\frac{u_{n}}{d N}\right|^{p} \\
& \leq \sum_{n} K_{n+1}^{-p} e^{-p \beta n} \int_{\varphi V_{n}}\left|\frac{d u_{n}}{d N}\right|^{p} \\
& \leq \sum^{p} A^{p} K_{n+1}^{-p} \\
& \leq \frac{s_{0}^{d-1}}{1000}
\end{aligned}
$$

by Lemma 7 , this is because when $Q \in \Re_{n+1} \backslash \Im_{n+1}$, there exists $Q^{\prime} \in \Im_{n}$ such that $Q^{\prime} \supset Q$ but

$$
\int_{\varphi Q}\left|\frac{d u_{n}}{d N}\right|^{p}>K_{n+1}^{p} e^{-p \beta n}
$$

by definition. And so

$$
|\varphi Q|<K_{n+1}^{-p} e^{-p \beta n} \int_{\varphi Q}\left|\frac{d u_{n}}{d N}\right|^{p} .
$$

Finally we get

$$
\left|\left\{X \in \varphi Q_{0}: u(X)=0, \nabla u(X)=0\right\}\right| \geq \frac{1}{100}\left|\varphi Q_{0}\right|>0 .
$$

\section{Proof of Theorem.}

Case 1: $D$ is a bounded domain. Then we can assume $(0,0) \in \partial D$ and $\mathbb{R}^{d-1}$ is the tangent space of $\partial D$ at $(0,0)$ and $D \subset \mathbb{R}_{+}^{d}$. So we may 
construct a domain $\Omega=\Omega_{\varphi}=\left\{\left(x, x_{d}\right): x_{d}>\varphi(x)\right\}$ where $\varphi \in \Phi_{2 M}$ and $D \subset \Omega$ and $\partial D \cap \partial \Omega \supset \varphi Q_{0}$ for some $Q_{0}=Q_{0}\left(0, s_{0}\right)$ with $s_{0}>0$. Then apply Theorem 1 to $\Omega$, we get a harmonic function $U$ in $\Omega$ which is $C^{1}$ up to $\bar{\Omega}$ such that

$$
\left|\left\{X \in \varphi Q_{0}: U(X)=0, \nabla U(X)=0\right\}\right|>0 .
$$

Now let $u=\left.U\right|_{D}$, then this is the desired $u$.

Case 2: $D=\mathbb{R}^{d} \backslash B$ for some bounded $C^{1, s}$ domain $B$. We assume that $(0,0) \in B$. Let $T: X \rightarrow X /|X|^{2}$ be the Kelvin transformation and $\tilde{B}=\left.\mathbb{R}^{d}\right|_{T B}$. Apply the result in Case 1 to $\tilde{B}$ and get a harmonic function $U$. Then $u(X)=U(T X) /|X|^{d-2}$ is the desired function for our domain $D$.

Case 3: $D$ is a general $C^{1, s}$ domain. It is easy to find a domain $B \subset \bar{D}^{c}$ such that $\partial B \cap D$ contains some "ball" on $\partial D$. Then by the Case 2, there is a harmonic function $U$ in $\mathbb{R}^{d} \backslash B$ which is $C^{1}$ up to $\partial B$ and $\mid\{X \in$ "ball" : $U=\nabla U=0\} \mid>0$. Then this $u(X)=\left.U\right|_{D}$ is needed.

Some Remarks. 1. The theorems are true for $C^{1}$-Dini domains. The proof follows our argumens with minor corrections.

2. It is not hard to see that for every $\varepsilon$ there exists a harmonic function which is $C^{1}$ up to the boundary and such that

$$
\mid \partial D \backslash\{X \in \partial D: u(X) \neq 0 \text { or } \nabla u(X) \neq 0\} \mid \leq \varepsilon .
$$

3. We do not know if the theorem is true or not for Lipschitz domains. In fact, our method does not work even for $C^{1}$ domains (and even if we do not need the restriction $u=0$ ).

4. We may also prove Lemma 4 by using the potential layer theory as in [2]. But again this method does not work even for $C^{1}$ domains.

Acknowledgments. I would like to express my thanks to my teacher and advisor, Proffesor T. Wolff, for bringing this problem to my attention as well as for explaining to me the idea in his paper [2]. 


\section{References.}

[1] Aleksandrov, A. and Kargaev, P., private communication with T. Wolff.

[2] Bourgain, J. and Wolff, T., A remark on gradients of harmonic functions in dimension $d \geq 3$. Colloq. Math. 40/41 (1990), 253-260.

[3] Caffarelli, L., Fabes, E. and Kenig, C. E., Completely singular elliptic harmonic measures. Indiana Univ. Math. J. 30 (1981), 917-924.

[4] Gruter, M. and Widman, K., The Green function for uniformly elliptic equations. Manuscripta Math. 37 (1982), 303-342.

[5] Kenig, C. E., Elliptic boundary value problems. Beijing Lectures in Harmonic Analysis ed. E. M. Stein, Ann. of Math. Studies 112, Princeton Univ. Press, 1986.

[6] Krantz, S., Function theory of several complex variables. John Wiley and sons, 1982.

Recibido: 30 de mayo de 1.993

Revisado: 18 de septiembre de 1.993

Wensheng Wang

Department of Mathematics

University of Southern California

Los Angeles CA 90089, U.S.A.

wang@math . us c.edu 